\title{
A Model of Organic Consumption
}

Haesun Park-Poaps, Morgan State University, Baltimore, MD.

Keywords: Organic, Environment, Sustainability, Consumption

During the last two decades, the market for organic products has grown rapidly and reached $\$ \$ 35.1$ billion in 2013 in the US, representing 11.5\% increase from the previous year. Growing consumers' interests toward health, animal welfare and environmental protection is the major driver for the market growth (Magnusson et.al, 2003; Saba \& Messina, 2003). Many studies of organic consumption have investigated the impacts of environmental and health concerns/beliefs and benefits of organic products. However, findings provide inconclusive evidence regarding the effects of the concerns/beliefs, especially when the actual purchase or decisions are investigated (Magnusson et al., 2003). Some studies have indicated that environmental and health risks maybe a more powerful, reliable predictor of organic consumption (Saba \& Messina, 2003). Perception that one's actions (e.g., organic purchase) in fact contribute to the environment has also been found to be a strong predictor of environmental behaviors (Lord \& Putrevu, 1998; Verhoef, 2005). There is a need to develop a theory of organic consumption that can be generalized across different organic product categories based on the perspectives of both risk perception and the efficacy.

The purpose of this study is to conceptualize organic consumption that applies to a wide range of consumer product categories, including food, household items, and fiber products. The conceptual model will include dimensions that shape the environmental risk and efficacy perceptions and their relationships with organic consumption.

Risk perception is a subjective judgment or estimation about a risk (or uncertain consequence). Human judgement or decisions involves risk perceptions that are not only cognitive but also intuitive in nature. Risk perceptions tend to be biased toward information that people are exposed to or experience. In addition to the probability of the risk, when people are presented with risk information, how risk information is presented/ framed (Tversky \& Kahneman, 1974) can be a more powerful predictor of risk reducing behaviors.

Further, there are judgmental discounters, sometimes seen as dimensions of 'framing effects' in information processing studies, which seem negate the risk consequences: 1) temporal delays, 2) spatial distance, and 3) target distance (Fleury-Bahi, 2008). First, discounting of the risk or outcome occurs when the consequence of the risk is delayed. People estimate the risks that occur in immediate future as being greater than the risks that have long-term consequences. Second, risks or outcomes that occur in distant places yield a similar discounting effect. People tend to attenuate risks that happen distant places. A large number of studies have found that when people perceive their environmental behaviors have positive consequences for themselves they tend to engage in environmental behaviors (e.g., vehicle usage, household recycling, and household supply and food consumption). The risk perceptions in the literature were primarily applied to uncertainty/probability and monetary loss or gain contexts in decision making. However, environmental outcomes share similar patterns of discounting (Guyse et al., 2002).

Perceived consumer effectiveness is defined as the consumers' belief that they, as individuals, can help solve [environmental] problems (Roberts, 1996, p. 217). Consumers who feel helpless to make any positive change in a social condition through their own behavior may be more susceptible to frustration and discomfort and may ignore the consequence of the social condition (Lord \& Putrevu, 1998). The concept of perceived consumer effectiveness is originated from moral decision making theories and scholars have used it to explain consumer 
environmental behaviors (e.g., energy use, recycling, organic product choice) (Lord \& Putrevu, 1998; Verhoef, 2005). It has been proven that perceived consumer effectiveness is a unique behavioral motivator, separate from the environmental concerns, that directly impacts behaviors (Lord \& Putrevu, 1998; Verhoef, 2005). In fact, studies found that attitudes toward proenvironmental behaviors or environmental concern predict actual behaviors only when perceived consumer effectiveness is included the relationship (e.g., Verhoef, 2005).

In a similar vein, trust in organic product claims can increase consumers' belief in the efficacy of organic products. Trust in organic production and processes increases consumers' belief about the effectiveness of organic products to solve environmental risks associated with conventional farming. On the other hand, skepticism about the product claims or organic certification system can undermine the value of organic products and discourages consumption. Consumers can be skeptical that products actually qualify for organic labeling. They might be skeptical of the certifying agencies or organic agriculture itself. When people lack knowledge or are uncertain about the risk/benefit at hand (skeptical), trust plays an essential part in risk/benefit perceptions (Siegrist \& Cvetkovich, 2000). Assured benefits from trusting organic production and their benefits are likely to aid decision making and corresponding actions (c.f., Siegrist \& Cvetkovich, 2000). Further, credibility/trust towards organic claims is likely to stimulate central processing of organic benefit information and thus influence behaviors.

The following visual model explains the interrelationships discussed and dimensions of the perceived risks. This study conceptualized organic consumption with the generalizable constructs, perceived environmental risks and consumer efficacy, incorporating intuitive and imperfect cognitive information processing perspectives. The model and an empirical test of the model will help building effective promotion strategies and consumer education strategies.

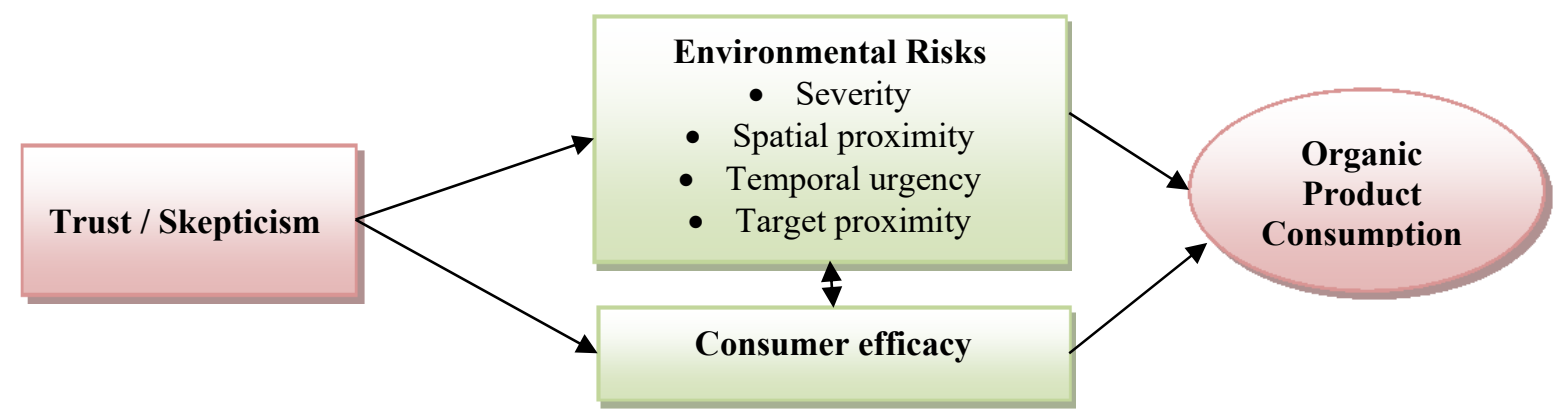

Fleury-Bahi, G. (2008). Environmental risk: perception and target with local versus global evaluation. Psychological Reports, 102(1), 185-193.

Guyse, J. L., Keller, L. R., \& Eppel, T. (2002). Valuing environmental outcomes: Preferences for constant or improving sequences. Organizational Behavior and Human Decision Processes, 87(2), 253-277.

Lord, K. R., \& Putrevu, S. (1998). Acceptance of Recycling Appeals: The Moderating Role of Perceived Consumer Effectiveness. Journal of Marketing Management, 14, 581-590.

Saba, A., \& Messina, F. (2003). Attitudes towards organic foods and risk/benefit perception associated with pesticides. Food Quality and Preference, 14(8), 637-645.

Siegrist, M., Cvetkovich, G., \& Roth, C. (2000). Salient values similarity, social trust, and risk/benefit perception. Risk Analysis, 20(3), 353-362.

Tversky, A. and Kahneman, D. (1974). Judgment under uncertainty: Heuristics and biases. Science, 185(4157), 1124-1131.

Verhoef, P. C. (2005). Explaining purchases of organic meat by Dutch consumers. Eur Rev Agric Econ, 32(2), 245-267. 